\title{
Autologous Mesenchymal Stem Cells Apceth_101
}

National Cancer Institute

\section{Source}

National Cancer Institute. Autologous Mesenchymal Stem Cells Apceth 101. NCI

Thesaurus. Code C113803.

Human autologous mesenchymal stem cells (MSCs) harvested from the bone marrow of a patient and genetically modified with a self-inactivating retroviral vector expressing the suicide gene herpes simplex virus thymidine kinase (HSV-TK), that can be used to activate synthetic acyclic guanosine analogues when co-administered. Upon intravenous administration of autologous mesenchymal stem cells apceth_101, the cells are actively recruited to the tumor stroma, differentiate into more mature mesenchymal cells, and become part of the tumor microenvironment. When a synthetic acyclic guanosine analogue, such as ganciclovir, is co-administered, the HSV-TK within the HSV-TKtransduced MSCs will monophosphorylate this prodrug. Subsequently the monophosphate form is further converted to the diphosphate form and then to its active triphosphate form by cellular kinases. The active form of ganciclovir kills the HSV-TKtransduced MSCs and leads to a bystander effect, which eliminates neighboring cancer cells. Therefore, synthetic acyclic guanosine analogues are activated only at the tumor site, which increases their local efficacy and reduces systemic toxicity. 